\title{
On the use of differential scanning calorimetry for thermal hazard assessment of new chemistry: Avoiding explosive mistakes
}

\author{
Sebastian P. Green, ${ }^{[a, b]}$ Katherine M. Wheelhouse, ${ }^{[c]}$ Andrew D. Payne, ${ }^{[d]}$ Jason P. Hallett, ${ }^{*[b]}$ \\ Philip W. Miller ${ }^{*[a]}$ and James A. Bull*[a] \\ [a] S. P. Green, Dr P. W. Miller and Dr J. A. Bull \\ Department of Chemistry, Imperial College London, Molecular Sciences Research Hub, White City Campus, Wood Lane, London W12 0BZ, UK \\ E-mail: j.bull@imperial.ac.uk, philip.miller@imperial.ac.uk \\ [b] S. P. Green and Prof J. P. Hallett \\ Department of Chemical Engineering, Imperial College London, South Kensington Campus, Exhibition Road, London SW7 2AZ, U.K \\ E-mail: j.hallett@imperial.ac.uk \\ [c] Dr K. M. Wheelhouse \\ Chemical Development, Product Development \& Supply, GlaxoSmithKline, GSK Medicines Research Centre, Gunnels Wood Road, Stevenage, \\ Hertfordshire SG1 2NY, U.K \\ [d] A. D. Payne \\ Process Safety, Clinical Supply Chain, GlaxoSmithKline, GSK Medicines Research Centre, Gunnels Wood Road, Stevenage, Hertfordshire SG1 2NY, U.K. \\ Supporting information for this article is given via a link at the end of the document.
}

\begin{abstract}
Differential scanning calorimetry (DSC) is increasingly used as evidence to support a favourable safety profile of novel chemistry, or to highlight the need for caution. DSC enables preliminary assessment of the thermal hazards of a potentially energetic compound. However, unlike other standard characterisation methods, which have well defined formats for reporting data, the current reporting of DSC results for thermal hazard assessment has shown concerning trends. Around half of all results in 2019 did not include experimental details required to replicate the procedure. Furthermore, analysis for thermal hazard assessment is often only conducted in unsealed crucibles, which could lead to misleading results and dangerously incorrect conclusions. We highlight the specific issues with DSC analysis of hazardous compounds currently in the organic chemistry literature and provide simple 'best practice' guidelines which will give chemists confidence in reported DSC results and the conclusions drawn from them.
\end{abstract}

The development of novel chemical reagents is an essential step in the advancement of synthetic methodology, access to new chemical space, and ultimately the discovery of new medicines and materials. Chemists have become acutely aware of the potential safety hazards of newly discovered reagents, and the impact it can have on their wider use and applications. In particular, a misunderstanding or misjudgement in the safety profile of a new compound or process can have serious unforeseen consequences. The use of differential scanning calorimetry (DSC) is becoming a common means for organic chemists to either support the favourable safety profile of their new reagent or process, or to evidence the need for caution. Inclusion and discussion of potential hazards is laudable and is one way of helping improve awareness and attitudes towards safety within the chemistry community, which can be deficient a claim particularly levelled towards academia. ${ }^{[1,2]}$

DSC is an invaluable tool for chemists, enabling a 'first look' at a potentially energetic material. Using small quantities (approximately $5 \mathrm{mg}$ ), a rapid assessment of the thermal stability and the energetic yield of a compound or reaction mixture can be obtained. ${ }^{[3,4]}$ DSC is considered a screening technique, with limited sensitivity, but the results are accurate and robust enough to determine process temperatures to avoid thermal decomposition (often using $T_{\mathrm{D} 24}$ : the temperature at which the time to maximum decomposition rate under adiabatic conditions is $24 \mathrm{~h}$ ), ${ }^{[5]}$ estimate the severity of an exotherm (often using the adiabatic temperature rise, $\Delta T_{\mathrm{ad}}$ ), ${ }^{[5]}$ and even make predictions whether impact sensitivity and explosivity are a concern by using the Yoshida correlation. ${ }^{[6]}$ Such data, particularly on novel or under-explored compounds and reagents, is informative to chemists wishing to employ new synthetic methods. There are other thermal hazard tests available, such as accelerating rate calorimetry (ARC), reaction calorimetry, impact sensitivity or explosivity tests, but none are as ubiquitous or simple to use as the humble DSC. Recently, there have been studies seeking to characterise and compare the thermal and process hazards of compound classes, such as peptide-coupling reagents, ${ }^{[7]}$ oxidisers, ${ }^{[8]} \mathrm{N}$-heterocyclic iodanes, ${ }^{[9]}$ and diazo compounds; ${ }^{[10]}$ or processes such as Pd-catalysed cross-coupling, ${ }^{[11,12]}$ TIPS$\mathrm{EBX}^{[13]}$ and $\mathrm{NaH}$ with DMF/DMSO/DMAc. ${ }^{[14]}$

In contrast to these in-depth studies, DSC data reported in the mainstream organic chemistry literature generally forms a small part of a much broader demonstration of the synthetic utility of a reagent or process, perhaps used to make cautious statements about the stability or hazardous nature of a reagent. Compared to the rigorous reporting standards in place for characterisation data, it seems that all too often the vital experimental details required to reproduce a DSC experiment are lacking. This may be a consequence of the lack of specific rules in place for publishing this type of data and a lack of specialist knowledge at the author, reviewer and publisher levels. Additionally, DSC experiments are frequently performed, or results interpreted, without adherence to best practices (Figure 1 ), possibly indicating a lack of familiarity with the technique as applied to thermal hazard assessment. As a consequence, common errors are made which could lead to the dangerously misleading conclusion that a new reagent is safer or does not exhibit a significant exotherm when this may not be correct. We previously reported on how the use of unsealed crucibles led to the incorrect conclusion that 2-azido-4,6-dimethoxy-1,3,5triazine (ADT) had no exothermic decomposition and was "intrinsically safe"; when in fact it is highly energetic. ${ }^{[15]}$ 
Practical.

- Use sealed, high-pressure (HP) rated crucibles.

- Avoid unsealed or 'hermetic' crucibles.

- Consider carefully the crucible materials of construction and interactions with

your chemistry - avoid non-inert metals (Al) and polymer O-rings.

- Do not rely on TGA or STA alone.

- For best results, use the slowest practical heating rate.

Reporting.

- Report the equipment type, heating rate and exact crucible type!

- Precisely define the metrics used (e.g. $T_{\text {onset }}$ ) to report thermal stability to avoid

confusion - is it extrapolated onset or left-limit onset?

- Exemplar DSC experimental can be found in references 7, 8, 10 and 13

Interpretation.

Avoid absolute statements of thermal stability, ie. "the compound is stable up to $T_{\text {onset }}$. Simply report the left-limit onset temperature $\left(T_{\text {onset }}\right)$.

- Take care with overlapping endothermic events, such as melting points.

-When making a comparison to existing results, compare like with like: use the

same experimental conditions and test reference compound in your setup

Figure 1. Key areas to consider when using DSC to assess new chemistry.

\section{The state of DSC in the literature in 2019: is it reproducible?}

To illustrate the current deficiency in experimental detail, we looked at every article containing the term "DSC" or "differential scanning" published in 2019 in a range of journals covering organic chemistry (Figure 2, see SI for more details). Limiting to the use of DSC to assess the thermal hazard of a new reagent or process gave a modest dataset of 53 articles (Figure 2a). Unsurprisingly, a significant proportion of the articles were published in Org. Process Res. Dev. (OPRD), a journal covering process chemistry in the fine chemical industries. This type of safety assessment is routinely performed where there is any concern from specialist process safety departments.
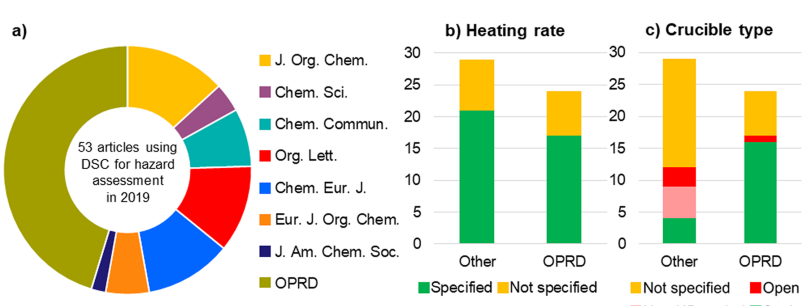

Figure 2. Reporting of DSC in the literature during 2019. a) Journal source of the 53 publications that used DSC for thermal hazard assessment in 2019. b) The number of publications that specified the heating rate used, divided into those from OPRD and from other journals. c) Publications specifying whether open or closed crucibles were used, from OPRD and other journals.

In a DSC experiment, the heating rate and the type of crucible used are two crucial factors. The heating rate is essential to the determination of the result and directly affects the temperature at which an exotherm is detected, as well as the exotherm peak shape and area, which affects the enthalpy measurement. ${ }^{[16]}$ As a result, any DSC thermal stability or $\Delta H_{D}$ experiment and result cannot be reproduced or compared without using the same heating rate. Surprisingly, approximately $30 \%$ of the articles from Figure $2 a$ did not specify the heating rate, both in OPRD and other journals (Figure 2b). In our experience, many literature reports prior to 2019 which are often cited as evidence for the hazardous nature of a compound (or lack thereof), also did not report sufficient experimental detail to enable the experiments to be replicated. Figure 3 illustrates an idealised exothermic DSC plot (Figure 3a) and summarises some concerning aspects observed in DSC plots in the recent literature, which are discussed in the following sections (Figure $3 b-d)$.
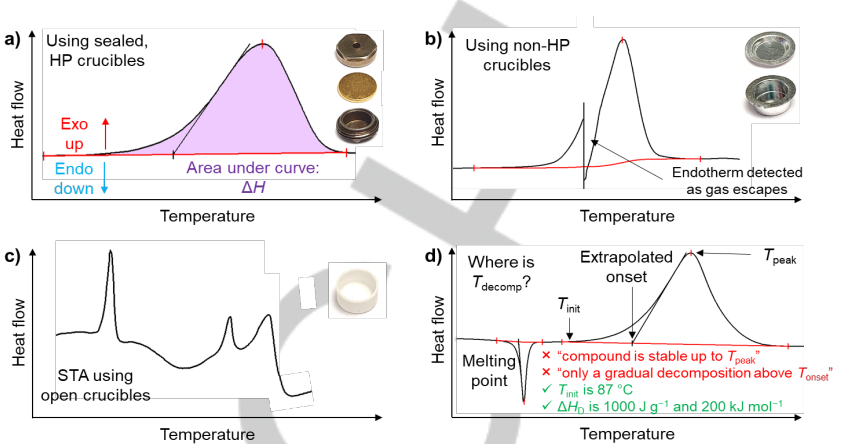

Figure 3. Representative sketches of DSC plots highlighting common errors observed in the literature. a) Ideal DSC plot of an exothermic decomposition using appropriate sealed HP crucibles, inset, which will more accurately represent the thermal stability and $\Delta H_{\mathrm{D}}$ (determined by calculating the shaded area under the peak) of a sample. b) Using typical hermetic or crimped $\mathrm{Al}$ pans, inset, which cannot withstand generated pressure leading to rupture and the sudden measurement of a large endotherm. c) Using STA, which requires open crucibles, such as the ceramic example inset, to measure mass loss leading to overlapping endothermic and exothermic events. d) Guidance on reporting thermal stability from a DSC experiment, showing incorrect statements we have observed in literature in 2019

\section{Measure something that is there!}

As an organic compound is heated in a DSC experiment, evaporation or loss of gaseous decomposition products is common, which registers as an endothermic event if an open crucible is used (Figure 4a). For analysing the thermally-induced decomposition of potential energetic materials, sealed highpressure (HP) rated crucibles are vital (Figure 4b; sealed glass ampoules have been shown to be an alternative $\left.{ }^{[17]}\right) \cdot{ }^{[4,18,19]}$ Hermetic (or crimped) aluminium crucibles are ubiquitous for other DSC measurements, such as phase transitions, and used open, with a pierced lid, or hermetically sealed with a press. These are inappropriate for hazard assessment however, as even when properly sealed they are incapable of withstanding much generated pressure and often rupture, causing a sudden endotherm (Figure 3b).
Evaporation

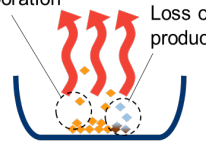

Open crucible: Heat escapes

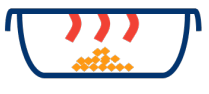

Sealed crucible: Heat detected

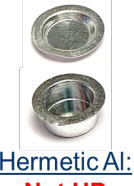

$\frac{\text { Hermetic Al: }}{\text { Not HP }}$

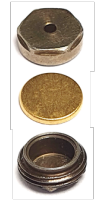

Inert metal:
Figure 4. Guidance on the types of DSC crucibles suitable for thermal hazard assessment. a) Evaporation of a sample or decomposition evolving gaseous products, upon escape from an open DSC crucible, may register as an endothermic event and give potentially misleading results. b) Disposable 'hermetic' AI DSC crucibles are inappropriate for thermal hazard assessment as they are not rated to withstand the pressures generated by mg quantities of samples. Alongside is an example of commercially available sealed, HP rated stainless steel crucibles (rated to 150 bar, reusable with disposable goldplated copper seal, PerkinElmer B0182901 and other brands are also available). Further detail on crucible selection is given in the SI. DSC 
equipment can be used for a variety of different experiments, for which different crucible types are appropriate.

With inappropriate crucibles, results may fail to reflect the true exothermic nature of an energetic material, which could result in the dangerously misleading conclusion that the compound is safe to use at temperatures where thermal runaway leading to an explosion could occur. ${ }^{[15]}$

Of the articles from OPRD in this dataset, the crucible type was specified in $70 \%$ (Figure 2c). Moreover, of those that did specify, all but one used a sealed HP crucible made from unreactive gold or tantalum-lined steel, stainless steel or a glass ampoule. In contrast, the crucible type was only specified in $41 \%$ of articles in this dataset from other journals (Figure $2 \mathrm{c}$ ). Of these 12 articles, 3 used Al crucibles with pierced lids (open crucibles); 3 used Al crucibles, 2 used hermetically sealed or crimped Al crucibles and only 4 used HP stainless steel or goldplated crucibles that we would consider suitable.

Selected examples that didn't specify the use of HP crucibles include studies on gem-diiodomethyl carbonyl compounds such as 1 (Figure 5a), ${ }^{[20]}$ pyridine-stabilised ketenimine salts, ${ }^{[21]}$ such as 2 (Figure $5 b$ ) and $N$-tosyl-4chlorobenzenesulfonimidoyl fluoride ${ }^{[22]}$ (SulfoxFluor, Figure 5c). In each case, these are presented as alternatives to more common reagents (cf diazo compounds, ketenimines, and nonaflyl fluoride or other fluoride sources respectively), demonstrating more desirable properties and DSC is used to support an improved stability or hazard profile for the new reagents. However, features of the DSC plots that were included suggest to us that evaporation or loss of gaseous decomposition products has affected the results: typically a minor exotherm that develops into a much larger endotherm as the temperature increases, obscuring any exothermic decomposition. ${ }^{[15]}$ Sealed HP crucibles would more accurately determine the thermal stability and exothermic behaviour of each compound.

As sealed crucibles are required to accurately capture exothermic events, thermogravimetric analysis (TGA) or simultaneous thermal analysis (STA, a combined DSC and TGA experiment) alone (both of which require open crucibles to measure mass loss) are not the most appropriate methods to measure the thermal stability and enthalpy of decomposition (see Figure 3c). Sharpless \& Dong recently reported a highly reactive diazo transfer reagent for the preparation azides from primary amines $\left(\mathrm{FSO}_{2} \mathrm{~N}_{3}\right.$, Figure $\left.5 \mathrm{~d}\right){ }^{[23]}$ This reagent was demonstrated in fragment synthesis and late stage functionalisation through the generation of a vast library of azides, themselves used for click cycloaddition to prepare triazoles. In the $\mathrm{SI}$, a detailed description of safety precautions for use is given alongside impact and friction sensitivity, toxicity, and other tests; and the authors were careful to advise against isolating the reagent. The authors also report STA data, the results of which exhibited large endothermic events as mass was lost from solutions of $\mathrm{FSO}_{2} \mathrm{~N}_{3}$ as material evaporated and gas-evolving decomposition ensued. Nonetheless, it would also be valuable to compare this low-molecular-weight sulfonyl azide to previously reported energetic sulfonyl azides if measured in sealed HP crucibles.
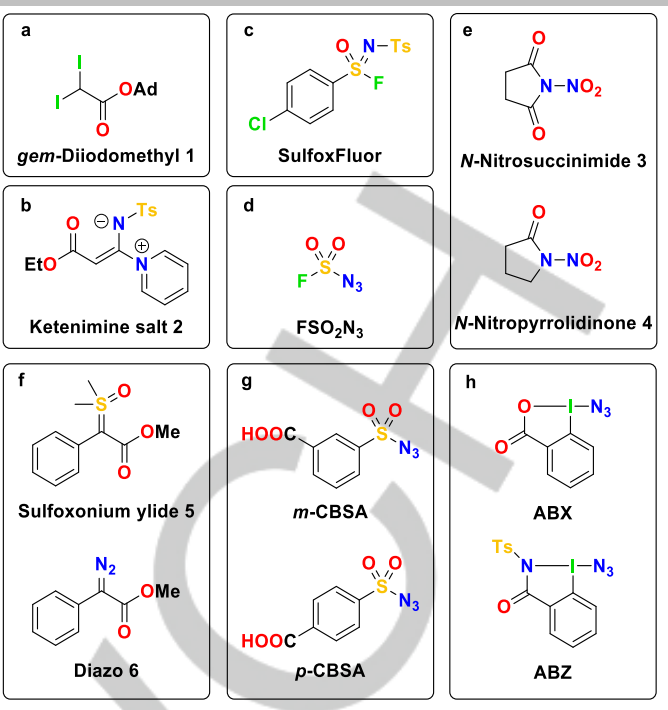

Figure 5. Selected examples where DSC was used to assess the thermal hazard of new chemistry. The functional groups present that typically contribute to an exothermic thermal decomposition are highlighted.

Similarly, novel radical nitrating reagent, $\mathrm{N}$-nitrosuccinimide 3 (Figure $5 \mathrm{e}$ ), was developed to transfer $\mathrm{NO}_{2}$ to alkenes, or form isoxazolines and isoxazoles from alkenes and alkynes respectively through photolytic formation of a nitryl radical species. ${ }^{[2]}$ Reagent $\mathbf{3}$ was selected over $N$-nitropyrrolidinone $\mathbf{4}$ and $N$-nitrophthalimide, among other reasons, for a preferable thermal decomposition profile evidenced by an STA plot. For two of these compounds, the DSC heat flow plots alongside steady mass loss in the TGA suggest concurrent exothermic decomposition and endothermic evaporation or gas loss events (inferred from the chemical structure as gas evolution appears feasible) as would be expected in the open crucibles used in STA. Sealed HP crucible DSC would prevent the confounding endotherms to enable a more accurate determination of thermal stability and exothermic potential.

\section{Compare apples with apples}

A comparison to literature results is often the benchmark for reporting new chemistry, considering product yields, atom economy, cost or myriad other data to quantify why new conditions are better than established techniques. Discussion of the improved stability or favourable safety profile of new reagents is a common cause for comparison to literature data. However, with regard to DSC, the frequent lack of experimental detail makes this very difficult. For example, the literature comparison between a sulfoxonium ylide 5 (Figure $5 \mathrm{f}$ ), ${ }^{[25]}$ and the analogous diazo compound $6,{ }^{[26]}$ is unreliable without detailing the controlled variables such as heating rate and crucible type, that directly affect the result.

Even better is to repeat analysis of the literature reagent using the same DSC equipment to make the comparison. In a selected example, Maguire \& Collins proposed $m$ carboxybenzenesulfonyl azide ( $m$-CBSA, Figure $5 \mathrm{~g}$ ) as a green, cost-effective diazo transfer reagent. ${ }^{[27]}$ They conducted DSC analysis following the precedent of Bollinger and Tuma, ${ }^{[28-30]}$ using HP crucibles, the same heating rate, and included analysis of one of the compounds in the original study. This allowed direct comparisons to be made between their results and the prior data. Full reporting of DSC experimental conditions will 
enable future data, generated under the same conditions, to be directly compared and increases the value of reporting DSC results.

In a similar manner, while a DSC result should be fundamentally repeatable, outliers are possible and repeats can be used for an additional level of rigour. Sperry and co-workers conducted a seminal thermal hazard assessment of peptide coupling reagents. ${ }^{[7]}$ DSC results for each compound were collected in triplicate, the mean reported, and any data point outside of a $10 \%$ difference from the mean repeated. Samples were also weighed before and after analysis to check for leaks during the test which may have reduced the observed exotherm. Another example from Waser and co-workers demonstrated safer alternatives to azidobenziodoxolone (ABX, Zhdankin reagent, Figure $5 \mathrm{~h}$ ), most notably $A B Z$ (Figure $5 \mathrm{~h}$ ), ${ }^{[1]}$ using mean DSC results from two measurements, alongside impact and friction sensitivity testing.

\section{Careful in conclusions}

A frequently encountered misconception is that an exothermic decomposition behaves like a melting point, and therefore the sample will be stable at any temperature below the exothermic event observed in the DSC experiment. However, decomposition is a chemical reaction, where the rate has an exponential reliance on temperature, from Arrhenius kinetics. ${ }^{[5]}$ The temperature at which an event is observed in calorimetric experiments varies significantly dependent on instrument sensitivity, heating rate, and sample mass. Additionally, there is no agreed convention or consensus to the labelling and reporting of thermal stability data, and so it should be made clear which of the following are used (see Figure $3 \mathrm{~d}$ ). The leftlimit onset is the lowest temperature at which an exotherm's heat flow is greater than the limit of detection of the DSC instrument (often noted as either $T_{\text {init }}$ or $T_{\text {onset }}$ ) and is most appropriate for process safety determinations. The extrapolated onset is determined by DSC software based on the steepest incline of the peak (often also noted as $T_{\text {onset}}$ ) and is useful for comparisons between compounds where the DSC baseline is sloped or left-limit onset is obscured. Stoessel described the concept of 'onset' and $T_{\mathrm{D} 24}$ as a 'safe operating temperature' which can be obtained from a single DSC experiment, in his comprehensive book (specifically pp. 286-295). ${ }^{[5]}$

Unfortunately, there are numerous examples of statements that claim stability up to or beyond the temperature at which decomposition is observed, some of which are illustrated in Figure 3d. There are even examples in the 2019 dataset that describe the compound as stable up to the observed $T_{\text {peak, }}$ where the exothermic decomposition is at the highest measured rate of heat release, and which should not be used for thermal stability determination.

\section{Conclusion}

Results obtained with DSC are undoubtedly of value as an initial exploration of stability and thermal hazard assessment. This data can be enough to inform safe operating conditions and encourage the use of an otherwise off-limits or unattractive reagent in laboratories. In our journey using DSC to describe the potential hazards of reagents we are working with, we have walked the same path and experienced the same pitfalls as others and have had to learn from these difficulties in an area we were not initially experienced in. We encourage the inclusion of these studies, but they must adhere to known best practices to avoid misleading results and potentially dangerous incorrect conclusions. Having examined the current usage of DSC in the literature, there is clear room for improvement: some conclusive statements that a reagent is safe, non-explosive or does not exothermically decompose are not supported by the data presented; and $45 \%$ of all articles were missing key experimental details which make replication of these experiments impossible, in direct conflict with the author guidelines of every journal in the study. The DSC analysis must be conducted with inert sealed HP crucibles instead of nonpressure resistant crucibles made from potentially reactive metal such as aluminium; and full experimental detail reported so the chemistry community can have confidence in the published results, and the conclusions drawn must be supported by the experiments conducted and the data presented. Although the discussion in this article may be common knowledge to many, particularly in the process industries, we believe that wider awareness of these guidelines would improve chemical safety and prevent conclusions regarding the safety of new chemistry which are at best misleading and at worst dangerous.

\section{Acknowledgements}

For financial support, we gratefully acknowledge GlaxoSmithKline, the Pharmacat Consortium and the EPSRC for iCASE studentship funding, and The Royal Society [University Research Fellowship (UF140161, to JAB) and Research Grants (RG150444 and RGFIEAI180031)].

Keywords: differential scanning calorimetry $\cdot$ safety $\bullet$ thermal hazard $\bullet$ organic chemistry $\bullet$ sustainable chemistry

[1] A. D. Ménard, J. F. Trant, Nat. Chem. 2020, 12, 17.

[2] L. E. Grabowski, S. R. Goode, J. Chem. Heal. Saf. 2016, 23, 30.

[3] D. J. Frurip, Org. Process Res. Dev. 2008, 12, 1287.

[4] D. J. Frurip, T. Elwell, Process Saf. Prog. 2007, 26, 51

[5] F. Stoessel, Thermal Safety of Chemical Processes, Wiley-VCH Verlag $\mathrm{GmbH}$, Weinheim, 2008.

[6] T. Yoshida, F. Yoshizawa, M. Itoh, T. Matsunaga, M. Watanabe, Kogyo Kayak. 1987, 48, 311. The Yoshida correlation offers a prediction of explosivity and impact sensitivity from a single DSC experiment, though is not a substitute for the corresponding specialist tests. Certain classes of compound may give unreliable results in the Yoshida correlation, such as strained compounds. For more recent application and discussion, see ref 7.

[7] J. B. Sperry, C. J. Minteer, J. Y. Tao, R. Johnson, R. Duzguner, M. Hawksworth, S. Oke, P. F. Richardson, R. Barnhart, D. R. Bill, R. A Giusto, J. D. Weaver, Org. Process Res. Dev. 2018, 22, 1262.

[8] M. A. Dallaston, C. J. Bettencourt, S. Chow, J. Gebhardt, J. Spangler, M. R. Johnston, C. Wall, J. S. Brusnahan, C. M. Williams, Chem. Eur. J. 2019, 25, 9614

[9] A. Boelke, Y. A. Vlasenko, M. S. Yusubov, B. J. Nachtsheim, P. S. Postnikov, Beilstein J. Org. Chem. 2019, 15, 2311.

[10] S. P. Green, K. M. Wheelhouse, A. D. Payne, J. P. Hallett, P. W. Miller, J. A. Bull, Org. Process Res. Dev. 2020, 24, 67.

[11] Q. Yang, N. Sane, D. Klosowski, M. Lee, T. Rosenthal, N. X. Wang, E. Wiensch, Org. Process Res. Dev. 2019, 23, 2148

[12] Q. Yang, N. R. Babij, S. Good, Org. Process Res. Dev. 2019, 23, 2608.

[13] D. P. Hari, P. Caramenti, L. Schouwey, M. Chang, S. Nicolai, D. Bachert, T. Wright, C. Orella, J. Waser, Org. Process Res. Dev. 2020, 24, 106.

[14] Q. Yang, M. Sheng, J. J. Henkelis, S. Tu, E. Wiensch, H. Zhang, Y Zhang, C. Tucker, D. E. Ejeh, Org. Process Res. Dev. 2019, 23, 2210. 
[15] S. P. Green, A. D. Payne, K. M. Wheelhouse, J. P. Hallett, P. W. Miller J. A. Bull, J. Org. Chem. 2019, 84, 5893.

[16] For a DSC experiment, the slowest practical heating rate will provide the most accurate thermal stability determination. This minimises any thermal lag effect observed in the results, as heat transfer between the sample and sensor is typically worse for crucibles with a thicker base, such as the HP crucibles recommended. A slower heating rate is particularly important for compounds with a rapid exotherm onset, which is often the case with energetic molecules.

[17] M. Sheng, D. Valco, C. Tucker, E. Cayo, T. Lopez, Org. Process Res. Dev. 2019, 23, 2200

[18] G. Hentze, Thermochim. Acta 1984, 72, 127.

[19] S. M. Rowe, Org. Process Res. Dev. 2002, 6, 877

[20] A. G. Herraiz, M. G. Suero, Chem. Sci. 2019, 10, 9374

[21] N. P. Massaro, A. Chatterji, I. Sharma, J. Org. Chem. 2019, 84, 13676.

[22] J. Guo, C. Kuang, J. Rong, L. Li, C. Ni, J. Hu, Chem. Eur. J. 2019, 25, 7259.

[23] G. Meng, T. Guo, T. Ma, J. Zhang, Y. Shen, K. B. Sharpless, J. Dong, Nature 2019, 574, 86.

[24] K. Zhang, B. Jelier, A. Passera, G. Jeschke, D. Katayev, Chem. Eur. J. 2019, 25, 12929 .

[25] C. Janot, P. Palamini, B. C. Dobson, J. Muir, C. Aïssa, Org. Lett. 2019 21, 296

[26] H. E. Bartrum, D. C. Blakemore, C. J. Moody, C. J. Hayes, Chem. Eur. J. 2011, 17, 9586.

[27] R. M. O'Mahony, C. M. Broderick, D. Lynch, S. G. Collins, A. R. Maguire, Tetrahedron Lett. 2018, 60, 35.

[28] G. G. Hazen, L. M. Weinstock, R. Connell, F. W. Bollinger, Synth Commun. 1981, 11, 947.

[29] L. D. Tuma, Thermochim. Acta 1994, 243, 161.

[30] F. W. Bollinger, L. D. Tuma, Synlett 1996, 1996, 407

[31] S. Alazet, J. Preindl, R. Simonet-Davin, S. Nicolai, A. Nanchen, T. Meyer, J. Waser, J. Org. Chem. 2018, 83, 12334.

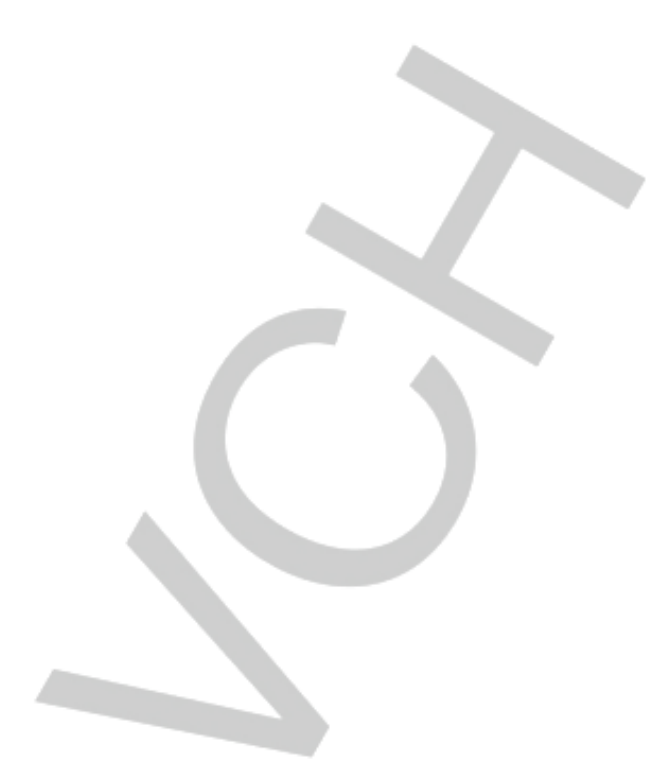




\section{Entry for the Table of Contents}

Insert graphic for Table of Contents here.

DSC for Thermal
Hazard Assessment

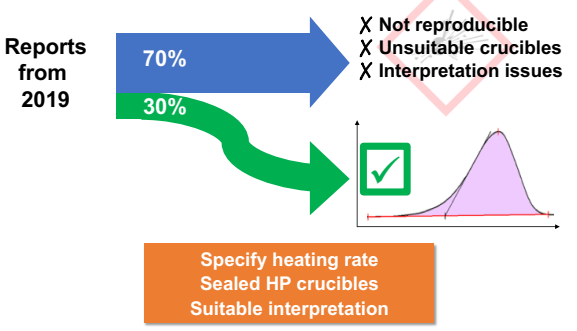

Analysis of the use of differential scanning calorimetry (DSC) for thermal hazard assessment in 2019 shows some alarming trends. Many reports are not reproducible, do not follow established best practices and the conclusions drawn are not supported by the data presented. This article discusses these issues and offer simple guidance on how to avoid them.

Institute and/or researcher Twitter usernames: @jamesabull, @HallettGroup, @PdCyclist, @SebPGreen, 